\title{
THE
}

2-27-2007

\section{Rigid Quantum Monte Carlo Simulations of Condensed Molecular Matter: Water Clusters in the $n=2$ - 8 Range}

Stephen F. Langley

E. Curotto

David L. Freeman

University of Rhode Island, dfreeman@uri.edu

J. D, Doll

Follow this and additional works at: https://digitalcommons.uri.edu/chm_facpubs

Terms of Use

All rights reserved under copyright.

\section{Citation/Publisher Attribution}

Langley, S. F., Curatto, E., Freeman, D. L., \& Doll, J. D. (2007). Rigid Quantum Monte Carlo Stimulations of Condensed Molecular Matter: Water Clusters in the $\mathrm{n}=2$ - > B Range. Journal of Chemical Physics, 126(8), 084506. doi: $10.1063 / 1.2484229$

Available at: http://dx.doi.org/10.1063/1.2484229

This Article is brought to you for free and open access by the Chemistry at DigitalCommons@URI. It has been accepted for inclusion in Chemistry Faculty Publications by an authorized administrator of DigitalCommons@URI. For more information, please contact digitalcommons-group@uri.edu. 


\title{
Rigid quantum Monte Carlo simulations of condensed molecular matter: Water clusters in the $n=2 \rightarrow 8$ range
}

\author{
Stephen F. Langley and E. Curotto ${ }^{\text {a) }}$ \\ Department of Chemistry, Arcadia University, Glenside, Pennsylvania 19038-3295 \\ and Department of Physics, Arcadia University, Glenside, Pennsylvania 19038-3295 \\ D. L. Freeman \\ Department of Chemistry, University of Rhode Island, Kingston, Rhode Island 02881-1966 \\ J. D. Doll \\ Department of Chemistry, Brown University, Providence, Rhode Island 02912-9127
}

(Received 8 November 2006; accepted 11 January 2007; published online 27 February 2007)

\begin{abstract}
The numerical advantage of quantum Monte Carlo simulations of rigid bodies relative to the flexible simulations is investigated for some simple systems. The results show that if high frequency modes in molecular condensed matter are predominantly in the ground state, the convergence of path integral simulations becomes nonuniform. Rigid body quantum parallel tempering simulations are necessary to accurately capture thermodynamic phenomena in the temperature range where the dynamics are influenced by intermolecular degrees of freedom; the stereographic projection path integral adapted for quantum simulations of asymmetric tops is a significantly more efficient strategy compared with Cartesian coordinate simulations for molecular condensed matter under these conditions. The reweighted random series approach for stereographic path integral Monte Carlo is refined and implemented for the quantum simulation of water clusters treated as an assembly of rigid asymmetric tops. (C) 2007 American Institute of Physics.
\end{abstract}

[DOI: $10.1063 / 1.2484229]$

\section{INTRODUCTION}

A number of recent investigations have contributed to the development, testing, and applications of path integral ${ }^{1-3}$ Monte Carlo methods ${ }^{4-25}$ in non-Euclidean manifolds ${ }^{26-28}$ arising in molecular condensed matter theory, whenever holonomic constraints are employed. Among the earliest attempts reported in the literature, one finds the simulation of bulk rigid water carried out in quaternion space with the fixed axis approximation. ${ }^{29}$ When there is a small number of linear rotors present in the system simulated, it is possible to split the kinetic energy operator in the density matrix expression. The angular momentum terms are isolated and then treated by vector space methods. ${ }^{30-34}$ Such an approach has been used to handle the simulation of a linear covalent molecule on surfaces, ${ }^{30,31}$ a linear molecule solvated by noble gas atoms, ${ }^{32-34}$ and para- $\mathrm{D}_{2}$, ortho- $\mathrm{D}_{2}$ clusters. ${ }^{35}$ Other authors have reported path integral Monte Carlo (PIMC) methods in torsional space using time slicing and angular variables. ${ }^{36,37}$ In a series of articles ${ }^{38-42}$ we have introduced and refined methodologies to carry out PIMC methods in rotational and torsional manifolds based on the DeWitt formula. ${ }^{2}$ We have established that torsional spaces and rotational spaces can be mapped using a general strategy; the maps are bijections from the manifold to an equidimensional Euclidean space. The stereographic projection coordinates extend from negative to positive infinity; therefore, once the metric of the manifold is transformed, it becomes possible to

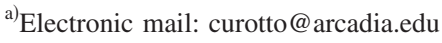

simulate the random walk using these "Cartesian-type" coordinates and to map the diffusion process back into the manifold. The one-to-one nature of the map between the angular variables and the stereographic projections is the key for the faithful representation of random paths in the manifold. In this manner, the notoriously difficult problem of evaluating the path integral inside open sets and the problem of imposing boundary conditions on the time evolution operator are avoided. Furthermore, using stereographic projections, it is possible to expand paths using a random series after generalizing the Feynman-Kaç formula in non-Euclidean spaces. This latter advance allows one to develop a fast converging algorithm for the PIMC method, without the need to evaluate the gradient or the Hessian of the potential. Additionally, one can develop efficient estimators based on numerical derivatives of the action; this development has been recently reported for path integral simulations with Cartesian coordinates by one of our groups. ${ }^{22,23}$ The extension of these techniques to non-Euclidean manifolds mapped with stereographic projections has been tested recently. ${ }^{42}$ Rigid hydrogen fluoride (HF) clusters from the dimer to the octamer have been simulated. ${ }^{42}$ Rigid diatomic molecules are linear rotors; however, these calculations would be intractable if the angular terms were treated with vector spaces.

The present article has two main purposes. Firstly, we investigate the efficiency gained in PIMC simulations of molecular condensed matter when holonomic constraints are used. Secondly, we make use of new improvements in the stereographic projection path integral algorithms applied to Cartesian products of inertia ellipsoid spaces to simulate wa- 
ter clusters of several sizes in the $100-500 \mathrm{~K}$ range. For the investigation of the efficiency gain, in particular, we are interested to learn how rigid body simulations are more efficient than flexible ones in quantum simulations. It is well known that rigid body simulations improve the efficiency of thermodynamic estimators in Metropolis simulations. However, the efficiency gained by treating high frequency modes rigidly has not been investigated carefully for quantum parallel tempering simulations. ${ }^{43-46}$ The gain in efficiency in classical Metropolis simulations of rigid bodies is usually quantifiable as a decrease in the statistical fluctuation at all the temperatures. ${ }^{47-49}$ We expect the same to hold for parallel tempering simulations. However, we anticipate that in quantum simulations the efficiency of the rigid body algorithms will have two contributions; the first should be a similar decrease in statistical fluctuations of the estimated thermodynamics properties, and the second, and probably more important contribution is the convergence with respect to the number of path variables of the properties, at temperatures where the high frequency modes are predominantly in the ground state. In this case, one expects the path integral Monte Carlo approach based on Cartesian coordinates to experience nonuniform convergence. Nonuniform convergence can be experienced even when highly efficient accelerating schemes, such as the reweighted random series (RRS) method, are employed in spaces mapped by Cartesian coordinates. In order to verify these statements, we carry out a number of numerical tests. We choose a coupled, harmonic, manyparticle one-dimensional system (a harmonic chain) to investigate the convergence issue with path integral simulations. The knowledge of the eigenvalues of the Hessian for the full system and its adiabatic limit allows us to use analytical formulas for the heat capacity as a function of $T$ and the Trotter number. Both primitive convergence and cubic convergence can be inspected analytically for the full system and its adiabatic limit. One can learn a great deal about the convergence behavior of the four estimators by inspecting graphs of the heat capacity as a function of the two variables. The harmonic chain is a reasonable yet simple model for condensed molecular matter, where the complications that require differential manifolds are avoided by restricting the motion to translations in one dimension only.

We carry out several rigid body quantum simulations for water clusters in the dimer through the octamer range.$^{50}$ For this purpose, we revisit the RRS method recently developed for non-Euclidean manifolds, and we find a simple but important improvement for its numerical implementation. We simulate a particle in a ring and a rigid water monomer, with its rotations hindered by an external potential field to test the new improvements.

The remainder of this article is organized as follows: The improved RRS stereographic projection path integral for simulations in non-Euclidean spaces is discussed in Sec. II. All the numerical tests are presented in Sec. III. Section IV contains our conclusions.

\section{METHODS}

The partial averaging method and RRS approach have been extended to non-Euclidean spaces in a series of previ- ous works. ${ }^{38-42}$ We revisit the basic theory in the present manuscript since a computationally more efficient approach is developed. We begin with the elements of the density matrix in non-Euclidean manifolds,

$\rho\left(q, q^{\prime}, \beta\right)=\int \mathcal{D} q \exp \left\{\mathcal{A}_{g}-\frac{1}{\hbar} \int_{0}^{\beta \hbar} d \tau\left[\frac{1}{2} g_{\mu \nu} \dot{q}^{\mu} \dot{q}^{\nu}+V\right]\right\}$,

where $\mathcal{A}_{g}$ contains the natural logarithm of the square root of the determinant of the metric tensor evaluated at every point along the path. To develop the equivalent of the FeynmanKaç formula in non-Euclidean spaces, we introduce a convenient reference space,

$$
\begin{aligned}
& \frac{\rho\left(q, q^{\prime}, \beta\right)}{\rho_{0}\left(q, q^{\prime}, \beta\right)} \\
& \quad=\frac{\int \mathcal{D} q \exp \left\{-(1 / \hbar) \int_{0}^{\beta \hbar} d \tau\left[\mathcal{L}^{(0)}+\Delta \mathcal{L}+V\right]\right\}}{\int \mathcal{D} q \exp \left\{-(1 / \hbar) \int_{0}^{\beta \hbar} d \tau \mathcal{L}^{(0)}\right\}} .
\end{aligned}
$$

The symbol $\mathcal{L}^{(0)}$ represents a potential-free Lagrangian for a system of particles constructed in a convenient reference space of equal dimensionality with a constant (i.e., configuration independent) metric tensor,

$$
\mathcal{L}^{(0)}=\frac{1}{2} g_{\mu \nu}^{(0)} \dot{q}^{\mu} \dot{q}^{\nu}
$$

The Lagrangian $\mathcal{L}^{(0)}$ is used to handle the tail portion of the path truncated by the primitive algorithm to improve convergence. The geometric interaction is defined as

$$
\Delta \mathcal{L}=-\frac{N}{2 \beta} \ln \left(\operatorname{det}\left(g_{\mu \nu}\right)\right)+\frac{1}{2}\left[g_{\mu \nu}-g_{\mu \nu}^{(0)}\right] \dot{q}^{\mu} \dot{q}^{\nu}
$$

On the right, it is assumed that a trapezoid quadrature with $N$ points is used to evaluate the integral in $u=\tau / \beta \hbar$ so that

$$
\begin{gathered}
-\frac{1}{\hbar} \int_{0}^{\beta \hbar} d \tau\left(-\frac{N}{2 \beta} \ln \left(\operatorname{det}\left(g_{\mu \nu}\right)\right)\right) \\
=\frac{N}{2} \int_{0}^{1} d u\left(\ln \left(\operatorname{det}\left(g_{\mu \nu}\right)\right)\right) \\
=\frac{1}{2} \sum_{i=1}^{N} \ln \left(\operatorname{det}\left(g_{\mu \nu}\right)\right)=\mathcal{A}_{g} .
\end{gathered}
$$

To develop a random series algorithm, the infinite dimensional Riemann integral is recast in terms of path variables $a_{k}^{\mu}$ defined by

$$
q^{\mu}(u)=q_{r}^{\mu}(u)+\hbar \beta^{1 / 2} \sum_{k=1}^{\infty} a_{k}^{\mu} \Lambda_{k}(u),
$$

where the functions $\Lambda_{k}(u)$ have the following property in the 
interval $[0,1]$ :

$$
\int_{0}^{1} d u \frac{d \Lambda_{k}(u)}{d u} \frac{d \Lambda_{k^{\prime}}(u)}{d u}=\delta_{k k^{\prime}},
$$

and $q_{r}^{\mu}(u)=q^{\mu}+\left[\left(q^{\mu}\right)^{\prime}-q^{\mu}\right] u$.
Simply truncating the series for $q^{\mu}(u)$ to the first $k_{m}$ terms produces the primitive algorithm. To improve convergence, corrections for $\Delta \mathcal{L}+V$ are added to treat the tail portion of the series. This convergence enhancement can be done in two ways: One develops a partial averaging approach or a RRS method. Both approaches start with the Jensen inequality,

$$
\frac{\rho\left(q, q^{\prime}, \beta\right)}{\rho_{0}\left(q, q^{\prime}, \beta\right)}=\frac{\int d[a] \exp \left\{-\beta \int_{0}^{1} d u \mathcal{L}^{(0)}\right\}\left\langle\exp \left\{-\beta \int_{0}^{1} d u[\Delta \mathcal{L}+V]\right\}\right\rangle_{a}}{\int d[a] \exp \left\{-\beta \int_{0}^{1} d u \mathcal{L}^{(0)}\right\}} \geqslant \frac{\int d[a] \exp \left\{-\beta \int_{0}^{1} d u \mathcal{L}^{(0)}\right\} \exp \left\{-\beta \int_{0}^{1} d u\langle[\Delta \mathcal{L}+V]\rangle_{a}\right\}}{\int d[a] \exp \left\{-\beta \int_{0}^{1} d u \mathcal{L}^{(0)}\right\}},
$$

where $d[a]=d a_{1} \cdots d a_{k_{m}}, d a_{k}=d a_{k}^{1} \wedge d a_{k}^{2} \wedge \cdots d a_{k}^{D}$, and the average \langle\rangle$_{a}$ is taken over the (infinite) truncated tail coefficients $\left(d[a]_{a}=d a_{k_{m}+1} d a_{k_{m}+2} \cdots\right)$,

$$
\langle[\Delta \mathcal{L}+V]\rangle_{a}=\frac{\int d[a]_{a} \exp \left\{-\beta \int_{0}^{1} d u \mathcal{L}^{(0)}\right\}[\Delta \mathcal{L}+V]}{\int d[a]_{a} \exp \left\{-\beta \int_{0}^{1} d u \mathcal{L}^{(0)}\right\}} .
$$

A constant metric tensor in the reference Lagrangian $\mathcal{L}^{(0)}$ and a $D$-dimensional Dirac-delta function allow one to evaluate all the integrals contained in $\langle[\Delta \mathcal{L}+V]\rangle_{a}$ analytically,

$$
\begin{aligned}
& \langle[\Delta \mathcal{L}+V]\rangle_{a} \\
& \quad=\frac{\int d z \exp \left\{-(1 / 2) \Gamma_{\mu \nu} z^{\mu} z^{\nu}\right\}[\Delta \mathcal{L}+V]\left(q_{a}+z\right)}{\int d z \exp \left\{-(1 / 2) \Gamma_{\mu \nu} z^{\mu} z^{\nu}\right\}},
\end{aligned}
$$

where $q_{a}^{\mu}=q_{0}^{\mu}+\sum_{k=1}^{k_{m}} a_{k}^{\mu} \Lambda_{k}(u)$ is the core path and

$$
\Gamma_{\mu \nu}=\frac{g_{\mu \nu}^{(0)}}{\beta \hbar^{2}}\left[u(1-u)-\sum_{k=1}^{k_{m}} \Lambda_{k}^{2}(u)\right]^{-1} .
$$

If one expands both $\Delta \mathcal{L}$ and $V$ about the core path, one obtains the gradient partial averaging method,

$$
\frac{\rho^{\mathrm{PA}}\left(q, q^{\prime}, \beta\right)}{\rho_{0}\left(q, q^{\prime}, \beta\right)}=\frac{\int d[a] \exp \left\{-\beta \int_{0}^{1} d u\left[\mathcal{L}^{(0)}+\Delta \mathcal{L}+V+{ }_{2}^{-} \Gamma^{\mu \nu} \partial_{\mu} \partial_{\nu}(\Delta \mathcal{L}+V)\right]\right\}}{\int d[a] \exp \left\{-\beta \int_{0}^{1} d u \mathcal{L}^{(0)}\right\}} .
$$

This method converges quadratically in non-Euclidean spaces, but it requires the Hessian of the potential, that of the metric tensor, and that of $\ln \left(\operatorname{det}\left(g_{\mu \nu}\right)\right)$. All the additional computations in the term $\partial_{\mu} \partial_{\nu}(\Delta \mathcal{L}+V)$ can be avoided with the RRS method.

To develop the RRS method, one introduces a finite expansion of the path with $k_{m}^{\prime}>k_{m}$ terms,

$$
\begin{aligned}
& \widetilde{q}^{\mu}(u)=q_{r}^{\mu}(u)+\hbar \beta^{1 / 2} \sum_{k=1}^{k_{m}} a_{k}^{\mu} \Lambda_{k}(u)+\hbar \beta^{1 / 2} \sum_{k=k_{m}+1}^{k_{m}^{\prime}} a_{k}^{\mu} \widetilde{\Lambda}_{k}(u), \\
& \frac{\rho^{\mathrm{RW}}\left(q, q^{\prime}, \beta\right)}{\rho_{0}\left(q, q^{\prime}, \beta\right)}=\frac{\int d[a]_{r} \exp \left\{-\beta \int_{0}^{1} d u \mathcal{L}^{(0)}\right\} \exp \left\{-\beta \int_{0}^{1} d u[\Delta \mathcal{L}+V]\right\}}{\int d[a]_{r} \exp \left\{-\beta \int_{0}^{1} d u \mathcal{L}^{(0)}\right\}},
\end{aligned}
$$

and constructs the functions $\widetilde{\Lambda}_{k}(u)$ so that the partial averaging expansion about the core path derived from the above is equal to the same derived with the infinite series. In Eq. (14) we define the volume element for the RRS path integral $d[a]_{r}=d a_{1} \cdots d a_{k_{m}^{\prime}}$. One repeats the steps starting from Eq. (8) through Eq. (11), but replaces the infinite tail series $\sum_{k=k_{m}+1}^{\infty} a_{k} \Lambda_{k}(u)$ with $\sum_{k=k_{m}+1}^{k_{m}^{\prime}} a_{k} \tilde{\Lambda}_{k}(u)$ everywhere to find the functions $\widetilde{\Lambda}_{k}(u)$ that best reproduce the convergence of the partial averaging method. The requirement for $\widetilde{\Lambda}_{k}(u)$ is easily derived,

$$
\sum_{k=k_{m}+1}^{k_{m}^{\prime}} \tilde{\Lambda}_{k}^{2}(u)=u(1-u)-\sum_{k=1}^{k_{m}} \Lambda_{k}^{2}(u) .
$$

So far, the theoretical treatment is identical to that employed in Euclidean spaces; for non-Euclidean spaces there 
remains one issue for the development of RRS algorithms: How does one compute the integral $\int_{0}^{1} d u[\Delta \mathcal{L}+V]$ using the RRS? One cannot use all $k_{m}^{\prime}$ terms to compute the action directly since the $u$ derivatives of the $\Lambda_{k}$ functions are not orthogonal to those of the $\widetilde{\Lambda}_{k}$ functions. Consequently, the desired cancellations between the terms in $\mathcal{L}^{(0)}$ and those in $\Delta \mathcal{L}$ do not take place. Our first approach to work around this problem has been to substitute for the geometric interaction $\Delta \mathcal{L}$ an expansion up to the quadratic term about the core path and to recombine the terms in $\mathcal{L}^{(0)}$ for all the elements of the core path.

Here, we consider a simpler approach where the three terms $\mathcal{L}^{(0)}+\Delta \mathcal{L}+V$ are simply recombined into a single exponent before the integration over $u$ is carried out. Then, the proper terms cancel automatically without invoking additional definitions or properties of the path basis functions. This simple observation leads to a substantially simpler cubically convergent method,

$$
\begin{aligned}
\frac{\rho^{\mathrm{RW}}\left(q, q^{\prime}, \beta\right)}{\rho_{0}\left(q, q^{\prime}, \beta\right)} & =\frac{\int d[a]_{r} \exp \left\{-\beta \int_{0}^{1} d u U\left(\widetilde{q}^{\mu}(u)\right)\right\}}{\int d[a]_{r} \exp \left\{-\beta \int_{0}^{1} d u \mathcal{L}^{(0)}\right\}} \\
U\left(\widetilde{q}^{\mu}(u)\right)= & -\frac{N}{2 \beta} \ln \left[\operatorname{det} g_{\mu \nu}\left(\widetilde{q}^{\mu}(u)\right)\right]+\frac{1}{2} g_{\mu \nu}\left(\widetilde{q}^{\mu}(u)\right) \dot{q}^{\mu} \dot{q}^{\nu} \\
& +V\left(\widetilde{q}^{\mu}(u)\right) .
\end{aligned}
$$

Translating Eq. (16) into an algorithm yields a substantial improvement over the previous approaches. The expression for the important sampling procedure no longer requires the Hessian of the metric tensor and its determinant. Furthermore, in evaluating canonical averages for estimators,

$$
\left\langle A\left(q, q^{\prime}\right)\right\rangle=\frac{\int d q g^{1 / 2} A\left(q, q^{\prime}\right) \rho^{\mathrm{RW}}\left(q, q^{\prime}, \beta\right)}{\int d q g^{1 / 2} \rho^{\mathrm{RW}}\left(q, q^{\prime}, \beta\right)},
$$

the reference metric tensor is no longer needed in the importance sampling expression. This can be seen by writing the diagonal element of the reference density matrix explicitly,

$$
\begin{aligned}
\rho_{0}\left(q, q^{\prime}, \beta\right)= & \left(\frac{1}{2 \pi}\right)^{N D / 2}\left(\hbar^{2} \beta\right)^{-D / 2} J_{\Lambda} \int d[a]_{r} \\
& \times \exp \left\{-\beta \int_{0}^{1} d u \mathcal{L}^{(0)}\right\},
\end{aligned}
$$

where

$$
\begin{aligned}
& J_{\Lambda}=\operatorname{det}\left(\mathbf{J}_{\Lambda} \mathbf{J}_{\Lambda}^{T}\right)^{1 / 2}, \\
& \left(J_{\Lambda}\right)_{i j}=\lambda_{i}\left(u_{j}\right),
\end{aligned}
$$

and we have used the following convenient notation:

$$
\lambda_{k}(u)=\left\{\begin{array}{cc}
\Lambda_{k}(u) & \left(1 \leqslant k \leqslant k_{m}\right) \\
\tilde{\Lambda}_{k}(u) & \left(k_{m}+1 \leqslant k \leqslant k_{m}^{\prime}\right) .
\end{array}\right.
$$

Therefore, for an $N$ point quadrature, the quantity $J$ associated with the change of variables, $d q_{i} \rightarrow d a_{k}$, is $J$ $=\left(\hbar^{2} \beta\right)^{k_{m}^{\prime} / 2} J_{\Lambda}$. Upon combining all these results, $\rho^{R W}$ becomes,

$$
\begin{aligned}
\rho^{\mathrm{RW}}\left(q, q^{\prime}, \beta\right)= & \left(\frac{1}{2 \pi}\right)^{N D / 2}\left(\hbar^{2} \beta\right)^{-D / 2} J_{\Lambda} \int d[a]_{r} \\
& \times \exp \left\{-\beta \int_{0}^{1} d u U\left(\widetilde{q}^{\mu}(u)\right)\right\} .
\end{aligned}
$$

This simple modification to the algorithm allows us to state precise conditions for the convergence properties. In analogy with the results that have been established rigorously in Euclidean spaces, it is now possible to state the conditions that $\Delta \mathcal{L}+V$ must satisfy in order for the RRS techniques to converge. Namely, $\Delta \mathcal{L}+V$ must be in the Kato class $K_{d}^{\text {loc }}$, meaning roughly that $\Delta \mathcal{L}+V$ cannot have "bad singularities" or the method does not converge. Furthermore, for the FourierWiener path integral, when the path functions are

$$
\begin{aligned}
& \Lambda_{k}(u)=\sqrt{\frac{2}{\pi^{2}}} \frac{\sin (k \pi u)}{k}, \\
& \tilde{\Lambda}_{k}(u)=f(u) \sqrt{\frac{2}{\pi^{2}}} \frac{\sin (k \pi u)}{k},
\end{aligned}
$$

and

$$
f(u)=\sqrt{\frac{u(1-u)-\sum_{k=1}^{k_{m}}\left(2 / \pi^{2} k^{2}\right) \sin ^{2}(k \pi u)}{\sum_{k=k_{m}}^{k_{m}^{\prime}}\left(2 / \pi^{2} k^{2}\right) \sin ^{2}(k \pi u)}},
$$

it is possible to find an expression for the cubic convergence constant in non-Euclidean manifolds,

$$
\begin{aligned}
\lim _{k_{m} \rightarrow \infty} & k_{m}^{3}\left[\frac{\rho\left(q, q^{\prime}, \beta\right)-\rho^{\mathrm{RW}}\left(q, q^{\prime}, \beta\right)}{\rho\left(q, q^{\prime}, \beta\right)}\right] \\
= & \frac{\hbar^{2} \beta^{3}}{15 \pi^{4}} g^{(0) \mu \nu}\left\{\partial_{\mu}[\Delta \mathcal{L}+V] \partial_{\nu}[\Delta \mathcal{L}+V]\right. \\
& \left.+\partial_{\mu}^{\prime}[\Delta \mathcal{L}+V] \partial_{\nu}^{\prime}[\Delta \mathcal{L}+V]\right\} .
\end{aligned}
$$

In this equation, $g^{(0) \mu \nu}$ plays the same role as the $m_{0, i}^{-1}$ in Eq. (84) of Ref. 19, and $\partial_{\mu}^{\prime}=\partial / \partial\left(q^{\mu}\right)^{\prime}$ is the gradient at the path end point $q^{\prime}$.

With the new developments presented here, the RRS methods in non-Euclidean differentiable manifolds are nearly identical to those that have been developed in Euclidean spaces mapped with Cartesian coordinates. There remain only some minor differences, dictated mainly by the general nature of the potential-free reference Lagrangian $\mathcal{L}^{(0)}$. We have chosen $g_{\mu \nu}^{(0)}$ to replicate as closely as possible the effective mass of the particles so that the potential-free reference Lagrangian is as close as possible to its equivalent in the equidimensional Euclidean space mapped with Cartesian coordinates. In the present version of the theory the reference metric tensor $g_{\mu \nu}^{(0)}$ constitutes the only difference between the RRS methods in non-Euclidean and those developed in Euclidean space mapped with Cartesian coordinates. For the latter ones, it is convenient to rescale the path coefficients $a_{k}$ by $\hbar \beta^{1 / 2} / m_{0, i}$. We follow the same practice with $\hbar$ and $\beta^{1 / 2}$ because the temperature rescaling proves crucial for the performance of the energy and heat capacity estimators, 


$$
\begin{aligned}
\langle E\rangle_{\beta}= & \frac{D}{2 \beta}+\left\langle\frac{\partial}{\partial \beta}\left[\beta \int_{0}^{1} d u U\left(\widetilde{q}^{\mu}(u)\right)\right]\right\rangle, \\
\frac{C_{V}}{k_{B}}= & \frac{D}{2}+\frac{D^{2}}{4}+D \beta\left\langle\frac{\partial}{\partial \beta}\left[\beta \int_{0}^{1} d \tau U\left(\tilde{q}^{\mu}(u)\right)\right]\right\rangle \\
& +\beta^{2}\left\langle\left\{\frac{\partial}{\partial \beta}\left[\beta \int_{0}^{1} d u U\left(\tilde{q}^{\mu}(u)\right)\right]\right\}^{2}\right\rangle \\
& -\beta^{2}\left\langle\frac{\partial^{2}}{\partial \beta^{2}}\left[\beta \int_{0}^{1} d u U\left(\tilde{q}^{\mu}(u)\right)\right]\right\rangle \\
& -\left\{-\frac{D}{2}-\beta\left\langle\frac{\partial}{\partial \beta}\left[\beta \int_{0}^{1} d u U\left(\widetilde{q}^{\mu}(u)\right)\right]\right\rangle\right\}^{2},
\end{aligned}
$$
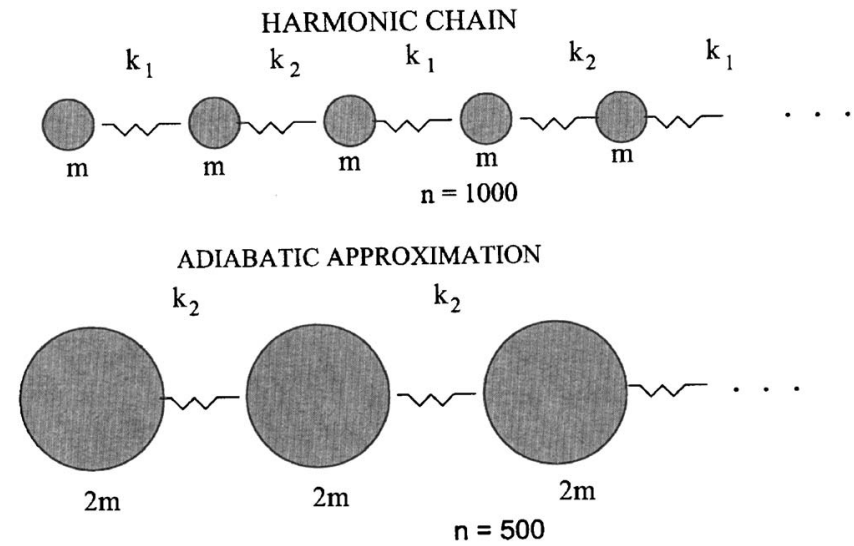

FIG. 1. A sketch of the harmonic chain. The point masses are represented by gray circles; these are allowed to vibrate about their equilibrium positions only in one dimension. In the top chain the spring constants alternate between the large value $k_{1}$, representing a intramolecular interaction, and the smaller value $k_{2}$, representing the intermolecular interaction. The bottom chain represents the adiabatic limit where the "diatomic molecules" represented by two point masses and the spring constant $k_{1}$ are condensed into a single point particle.

$$
\mathbf{H}=\frac{1}{m}\left(\begin{array}{ccccc}
k_{1} & -k_{1} & 0 & 0 & \\
-k_{1} & k_{1}+k_{2} & -k_{2} & 0 & \\
0 & -k_{2} & k_{1}+k_{2} & -k_{1} & \cdots \\
0 & 0 & -k_{1} & k_{1}+k_{2} & \\
& & \vdots & & \ddots
\end{array}\right) .
$$

The adiabatic approximation for the model is sketched at the bottom half of Fig. 1. Each pair of point masses connected by a spring with constant $k_{1}$ is now replaced by a $2 m$ point mass; each of these is connected to its neighbor(s) by an ideal spring with constant $k_{2}$. The mass-weighted Hessian is now $[n / 2] \times[n / 2]$ in dimension,

$$
\mathbf{H}^{(a)}=\frac{1}{2 m}\left(\begin{array}{ccccc}
k_{2} & -k_{2} & 0 & 0 & \\
-k_{2} & 2 k_{2} & -k_{2} & 0 & \\
0 & -k_{2} & 2 k_{2} & -k_{2} & \cdots \\
0 & 0 & -k_{2} & 2 k_{2} & \\
& & \vdots & & \ddots
\end{array}\right) .
$$

Figure 2 contains the eigenvalues of the flexible harmonic chain with $n=1000$ point masses for $k_{1}=0.1$ a.u. and $k_{2}=0.001$ a.u. The eigenfrequencies $\omega_{i}$ are sorted in ascending order, scaled by the square root of the mass, and plotted against the sorting index. The eigenfrequencies $\omega_{i}$ of the "intermolecular" degrees of freedom $(i \leqslant 500)$ are on left hand side of the graph in Fig. 2; those for the intramolecular degrees of freedom cases are on the right. The value of $\omega_{i}$ are discrete because the size $n$ is finite. However, there is a gap much larger than usual between $\omega_{501}$ and $\omega_{500}$. With close inspection of Fig. 2, one can easily verify that $\omega_{i} \approx \omega_{h}$ $=\sqrt{2 k_{1} / m}$, for $500<i \leqslant 1000$, whereas $\omega_{i} \rightarrow \omega^{*}=\sqrt{2 k_{2} / m}$ as $i \rightarrow 500$. The adiabatic limit produces a graph in the $1 \leqslant i$ $\leqslant 500$ range (white squares) that is indistinguishable from the flexible case in the same index range on the scale of Fig. 2. 


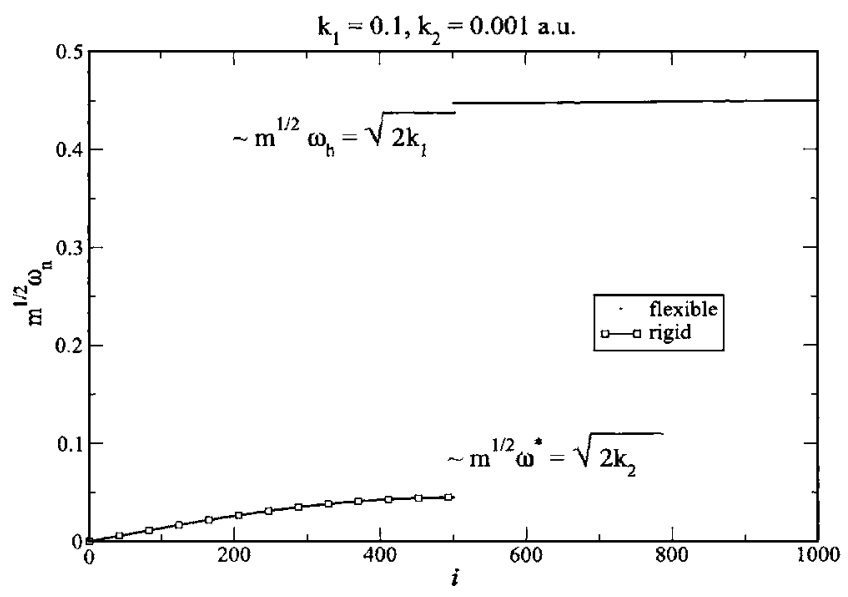

FIG. 2. The eigenvalues for the mechanical system depicted in Fig. 1, for $k_{1}=0.1$ a.u. and $k_{2}=0.001$ a.u., sorted in ascending frequencies.

\section{The $O(N)$ convergence of the quantum heat capacity}

Once the frequencies are computed from the eigenvalues of the Hessian, one can study the convergence of the path integral estimators using analytical formulas. Since the problem is reduced to a set of $n$ independent oscillators, it is simple to generalize well known analytical expressions for the thermodynamic properties at finite $N$ of a single harmonic oscillator. $N$ is the Trotter number or the number of random series coefficients. There are several questions that can be explored quickly with the simple harmonic chain. The first three of the following four questions, in particular, are important for the present discussion: How do the thermodynamic properties converge with the adiabatic approximation compared to the unconstrained counterpart? How much numerical advantage does the adiabatic approximation yield at cold temperatures? How does the numerical advantage gained by using constraints change for algorithms with improved convergence relative to the linear (or primitive) one? Does the size of the chain impact the convergence properties? To answer these questions we use the frequency sets $\left\{\omega_{i}\right\}_{i=1}^{n}$ for the flexible harmonic chain and $\left\{\omega_{i}^{a}\right\}_{i=1}^{n / 2}$ for the adiabatic approximation to calculate the heat capacity of the chain at several temperatures. To simulate linear convergence on $C_{V} / k_{B}$, we use the following expression derived from the discretized path integral with $N$ slices,

$$
\begin{aligned}
\left(\frac{C_{V}}{k_{B}}\right)_{N}= & \sum_{i} \frac{1}{4}\left(\beta \hbar \omega_{i}\right)^{2}\left(\frac{2}{\widetilde{\omega}_{i}+\widetilde{\omega}_{i}^{-1}}\right)^{2} \\
& \times\left\{\left(\frac{2}{\widetilde{\omega}_{i}^{N+1}+\widetilde{\omega}_{i}^{-(N+1)}}\right)^{2}\right. \\
& \left.+\frac{1}{N+1}\left[\frac{\widetilde{\omega}_{i}^{N+1}+\widetilde{\omega}_{i}^{-(N+1)}}{\widetilde{\omega}_{i}^{N+1}-\widetilde{\omega}_{i}^{-(N+1)}}\right]\left(\frac{\widetilde{\omega}_{i}-\widetilde{\omega}_{i}^{-1}}{\widetilde{\omega}_{i}+\widetilde{\omega}_{i}^{-1}}\right)^{2}\right\},
\end{aligned}
$$

where

$$
\widetilde{\omega}_{i}=\frac{1}{2} \frac{\beta \hbar \omega_{i}}{N+1}+\sqrt{1+\frac{1}{4}\left(\frac{\beta \hbar \omega_{i}}{N+1}\right)^{2}} .
$$

For the following discussion it is convenient to introduce two dimensionless temperature scales,

$$
T^{*}=\frac{1}{\beta \hbar \sqrt{2 k_{2} / m}}, \quad T^{\dagger}=\frac{1}{\beta \hbar \sqrt{2 k_{1} / m}} .
$$

Let us consider the case for the harmonic chain with $m=1$, $k_{1}=0.1$ a.u., $k_{2}=0.001$ a.u. For this case, the reader should note that $T^{\dagger}=T^{*} / 10$. Figure 3 contains graphs of the function in Eq. (32) plotted versus $T^{*}$ for several values of $N$. The left graph contains several adiabatic cases for different values of $N$, except for the set of points connected by the heavy dashed line; these correspond to the converged quantum result. The graph on the right contains plots for different values of $N$ for the flexible chain. The results in Fig. 3 demonstrate that the adiabatic approximation yields a substantial numerical advantage while remaining very accurate in the $0.1 \leqslant T^{*} \leqslant 1$ range; in this, the adiabatic approximation can compute $C_{V} / k_{B}$ with $N=16$, whereas the flexible computation needs a much greater number of slices (or path variables) to achieve a comparable convergence.
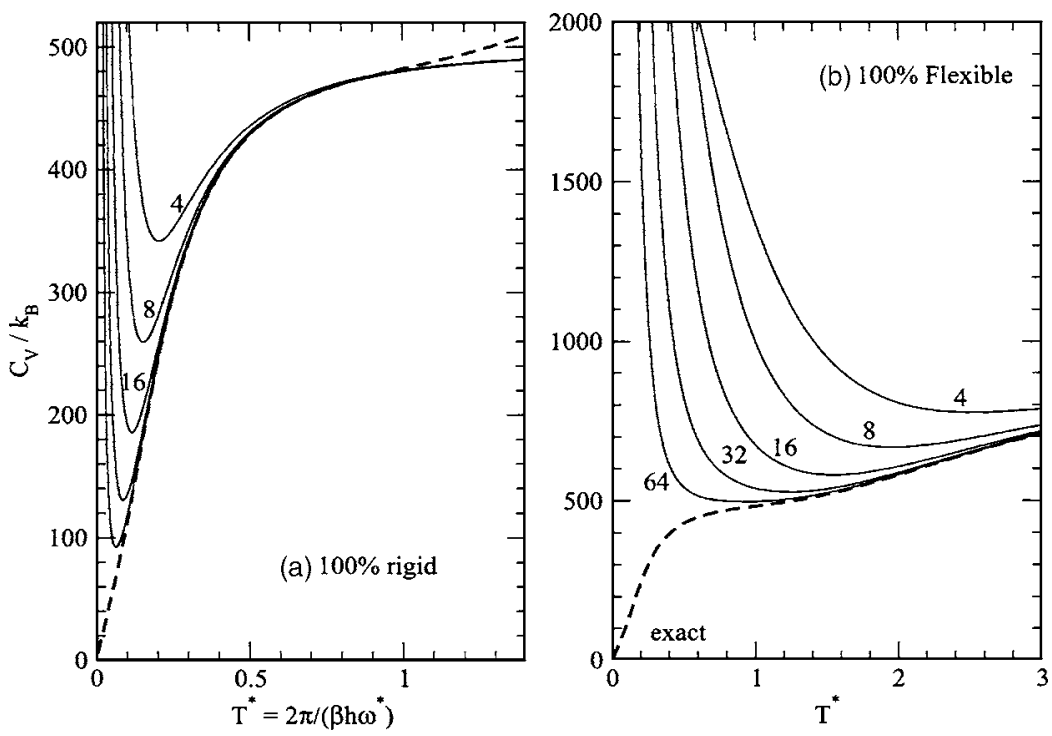

FIG. 3. The finite Trotter number $N$ heat capacity computed with the linearly convergent estimator in Eq. (32) and compared against the exact value (heavy dashed line). (a) is computed with the adiabatic set of frequencies; (b) is computed using all 1000 eigenvalues of the mechanical system depicted in Fig. 1 with $k_{1}=0.1$ a.u. and $k_{2}=0.001$ a.u.. 

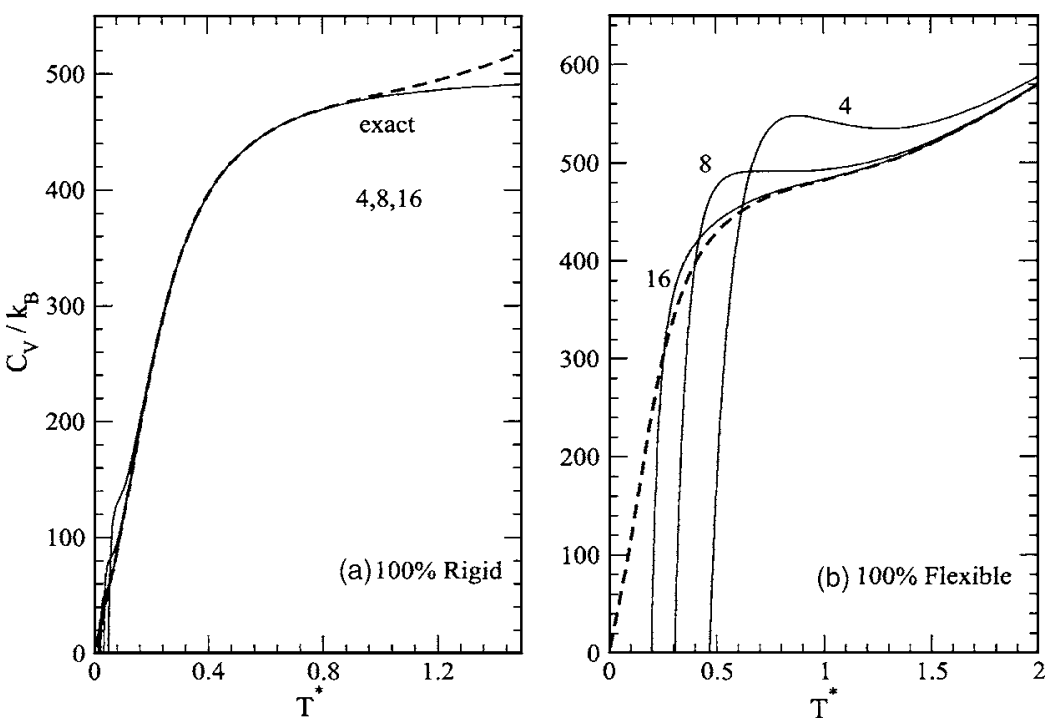

FIG. 4. The finite Trotter number $N$ heat capacity using (a) the adiabatic limit or (b) the entire set of eigenvalues for the heat capacity of the mechanical system depicted in Fig. 1 computed with the cubically convergent estimator in Eq. (35)

\section{The convergence of a higher order quantum heat capacity estimator}

The results obtained with Eq. (32) are compared with those obtained with the Fourier path integral $T$ - $H$ estimator since its analytical expression for the harmonic oscillator displays cubic convergence. The finite $N, T-H$ heat capacity estimator for the harmonic chain is

$$
\begin{aligned}
\left(\frac{C_{V}}{k_{B}}\right)_{N}= & \sum_{i}\left\{\frac{1}{2}-\frac{\beta \hbar \omega_{i}}{24}+\gamma_{i}+\frac{1}{2\left(1-8 \alpha_{i}\right)}\right. \\
& \left.-\frac{8\left(\alpha_{i}-\eta_{i}\right)}{\left(1-8 \alpha_{i}\right)^{2}}\right\},
\end{aligned}
$$

where $\alpha_{i}, \gamma_{i}$, and $\eta_{i}$ are the following sums over the random series coefficient index $k$ :

$$
\begin{aligned}
\alpha_{i} & =\sum_{k=1 \text { (odd) }}^{N} \frac{\left(\beta \hbar \omega_{i} / k \pi\right)^{2}}{(k \pi)^{2}+\left(\beta \hbar \omega_{i}\right)^{2}}, \\
\eta_{i}= & \sum_{k=1 \text { (odd) }}^{N}\left(\frac{\beta \hbar \omega_{i}}{k \pi}\right)^{2} \frac{\left(\beta \hbar \omega_{i}\right)^{2}}{\left[(k \pi)^{2}+\left(\beta \hbar \omega_{i}\right)^{2}\right]^{2}}, \\
\gamma_{i}= & \sum_{k=2 \text { (even) }}^{N}\left\{\left(\frac{\beta \hbar \omega_{i}}{k \pi}\right)^{2} \frac{1}{(k \pi)^{2}+\left(\beta h \omega_{i}\right)^{2}}\right. \\
& \left.+\frac{2}{\left[(k \pi)^{2}+\left(\beta \hbar \omega_{i}\right)^{2}\right]^{2}}\right\} .
\end{aligned}
$$

A set of curves similar to those in Fig. 3 is produced with Eq. (35) and plotted in Fig. 4. The superiority of the $T-H$ estimator is clear when one compares the curves in Fig. 4 with their counterparts in Fig. 3 for both the flexible and rigid cases. The numerical advantage of the adiabatic approximation in the $0.1 \leqslant T^{*} \leqslant 1$ range remains clear even when convergence is accelerated.

\section{B. The particle in a ring and the hindered rotations of a rigid asymmetric top}

A number of successful numerical tests have been carried out using the primitive Fourier path integral with projection coordinates in ellipsoids of inertia in earlier works. ${ }^{39}$ The testing of every part of an algorithm that performs path integration over a manifold composed of Cartesian products of many inertia ellipsoids with the RRS is a complex task. It is productive to brake down such algorithms by testing the theory gradually with systems of increasing complexity. We use the monodimensional non-Euclidean space $S^{1}$ to verify that the improved RRS method converges with better properties than the corresponding primitive algorithm. The particle in a unit radius ring with 207 a.u. of mass is subjected to a sinusoidal potential,

$$
V=\frac{4 \xi}{\xi^{2}+4}=\cos \theta
$$

where $0<\theta<2 \pi$ is the angular coordinate and $\xi$ is the stereographic projection. The set of points in the $x-y$ plane that are in the ring centered at the origin is mapped with the following equations:

$$
x=\frac{4 R^{2} \xi}{(\xi)^{2}+4 R^{2}}, \quad y=R \frac{(\xi)^{2}-4 R^{2}}{(\xi)^{2}+4 R^{2}} .
$$

The variable $\xi$ is obtained from the point $p \in \mathrm{S}^{1}$ by projecting with a straight line from the point $(0, \mathrm{R})$ through $p$ onto line $y=-R$. The map in Eq. (40) follows from simple geometric arguments. Two PIMC simulations aimed at testing Eqs. (23), (28), and (29) are carried out; one simulation uses $k_{m}^{\prime}$ $=k_{m}$ corresponding to the primitive algorithm and the other uses $k_{m}^{\prime}=4 k_{m}$ for the RRS. Both simulations use $k_{m}=8$ and are comprised of $1 \times 10^{6}$ "warmup" moves followed by 1 $\times 10^{6}$ moves over which data points are collected. Both simulations are repeated independently ten times to estimate the statistical error. The statistical error is estimated from the standard deviation to obtain a $95 \%$ confidence interval. In Fig. 5 the heat capacity of the particle in a ring computed with the primitive algorithm is compared with the same 


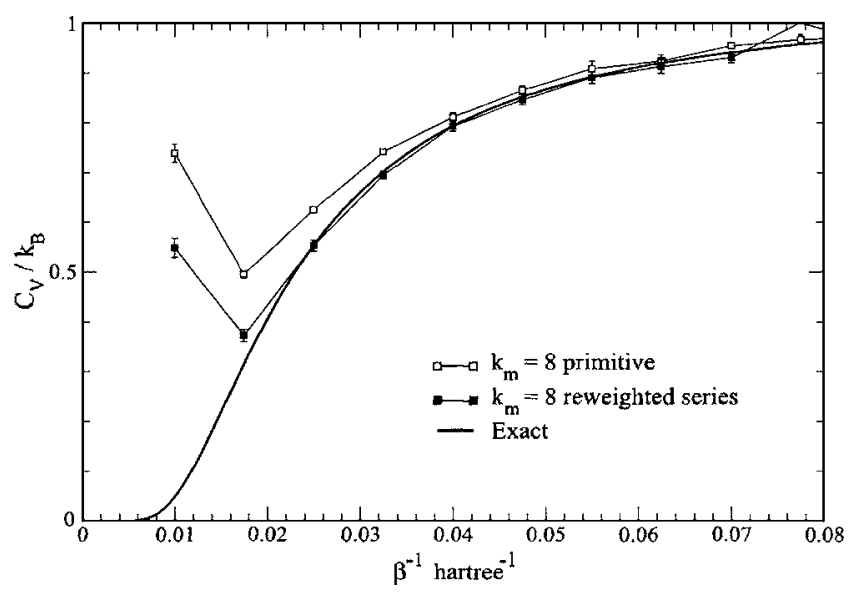

FIG. 5. The finite $k_{m}$ heat capacity for a particle with 207 a.u. of mass trapped in a ring with a $1.0 \mathrm{bohr}$ radius subjected to a $\cos \theta$ potential. The primitive algorithm (white squares) is compared with the improved RRS method (black squares) and with the exact result (heavy line).

quantity computed with the RRS method and with the exact result obtained by diagonalization. The particle in a ring is a simple and convenient system that has proved essential in testing new PIMC methods in non-Euclidean spaces.

Additional numerical tests are performed in the threedimensional inertia ellipsoid. $I_{\lambda}(\lambda=1,2,3)$ represents the eigenvalues of the moment of inertia tensor; the appropriate metric tensor for a rigid top is 39

$$
g_{\mu \nu}=I_{\lambda} J_{\mu}^{\mu^{\prime}} J_{\nu}^{\nu^{\prime}} f_{\mu^{\prime} \nu^{\prime}}^{\lambda}
$$

where the symbol $f_{\mu \nu}^{\lambda}$ represents a set of 18 independent functions obtained by writing the kinetic energy of a rigid asymmetric top in the laboratory frame in terms of the Euler angles $\theta, \phi$, and $\psi$. The symbol $J_{\mu}^{\mu^{\prime}}$ represents the Jacobian matrix element for the transformation between the Euler angle $q^{\mu^{\prime}}$ and the stereographic projection $q^{\mu}$. The $\xi^{1}, \xi^{2}, \xi^{3}$ $\rightarrow \theta, \phi, \psi$ map is ${ }^{39}$

$$
\begin{aligned}
& \theta=2 \sin ^{-1} \sqrt{\frac{\left(4 \xi^{2}\right)^{2}+\left(4 \xi^{3}\right)^{2}}{\left(\xi^{1}\right)^{2}+\left(\xi^{2}\right)^{2}+\left(\xi^{3}\right)^{2}+4}}, \\
& \phi=\tan ^{-1}\left(\frac{\left(\xi^{1}\right)^{2}+\left(\xi^{2}\right)^{2}+\left(\xi^{3}\right)^{2}-4}{4 \xi^{1}}\right)-\tan ^{-1}\left(\frac{\xi^{3}}{\xi^{2}}\right), \\
& \psi=\tan ^{-1}\left(\frac{\left(\xi^{1}\right)^{2}+\left(\xi^{2}\right)^{2}+\left(\xi^{3}\right)^{2}-4}{4 \xi^{1}}\right)+\tan ^{-1}\left(\frac{\xi^{3}}{\xi^{2}}\right) .
\end{aligned}
$$

These expressions can be easily inverted,

$$
\begin{aligned}
& \xi^{1}=\cos \frac{\theta}{2} \cos \frac{\phi+\psi}{2}\left(\frac{1+\cos (\theta / 2) \sin [(\phi+\psi) / 2]}{1-\cos (\theta / 2) \sin [(\phi+\psi) / 2]}+1\right), \\
& \xi^{2}=\sin \frac{\theta}{2} \cos \frac{\phi-\psi}{2}\left(\frac{1+\cos (\theta / 2) \sin [(\phi+\psi) / 2]}{1-\cos (\theta / 2) \sin [(\phi+\psi) / 2]}+1\right),
\end{aligned}
$$

$$
\xi^{3}=\sin \frac{\theta}{2} \sin \frac{\phi-\psi}{2}\left(\frac{1+\cos (\theta / 2) \sin [(\phi+\psi) / 2]}{1-\cos (\theta / 2) \sin [(\phi+\psi) / 2]}+1\right)
$$

We use geometric arguments similar to those for $\mathrm{S}^{1}$ to derive the map and its inverse from Eulerian angles to the stereographic projections by using the four-dimensional quaternion space and the spherical constraint. To simplify the notation further we introduce seven auxiliary quantities,

$$
\begin{aligned}
& d^{1}=\sqrt{16\left(\xi^{1}\right)^{2}+\left[\left(\xi^{1}\right)^{2}+\left(\xi^{2}\right)^{2}+\left(\xi^{3}\right)^{2}-4\right]^{2}}, \\
& d^{2}=\sqrt{\left(\xi^{2}\right)^{2}+\left(\xi^{3}\right)^{2}}, \\
& d^{3}=\left(d^{2}\right)^{2}, \quad d^{4}=\left(d^{1}\right)^{2}, \\
& d^{5}=8\left(\xi^{1}\right)^{2}-4\left[\left(\xi^{1}\right)^{2}+\left(\xi^{2}\right)^{2}+\left(\xi^{3}\right)^{2}-4\right], \\
& d^{6}=\left(\xi^{1}\right)^{2}+\left(\xi^{2}\right)^{2}+\left(\xi^{3}\right)^{2}+4, \\
& d^{7}=\left(\xi^{1}\right)^{2}+\left(\xi^{2}\right)^{2}+\left(\xi^{3}\right)^{2}-4 .
\end{aligned}
$$

Then, the Jacobian is

$$
J_{\mu^{\prime}}^{\mu}=\left(\begin{array}{c|c|c}
-\frac{16 d^{2} \xi^{1}}{d^{1} d^{6}} & \frac{2 d^{5} \xi^{2}}{d^{1} d^{2} d^{6}} & \frac{2 d^{5} \xi^{3}}{d^{1} d^{2} d^{6}} \\
\frac{d^{5}}{d^{4}} & \frac{8 \xi^{1} \xi^{2}}{d^{4}}+\frac{\xi^{3}}{d^{3}} & \frac{8 \xi^{1} \xi^{3}}{d^{4}}-\frac{\xi^{2}}{d^{3}} \\
\frac{d^{5}}{d^{4}} & \frac{8 \xi^{1} \xi^{2}}{d^{4}}-\frac{\xi^{3}}{d^{3}} & \frac{8 \xi^{1} \xi^{3}}{d^{4}}+\frac{\xi^{2}}{d^{3}}
\end{array}\right),
$$

while the nonvanishing $f_{\mu^{\prime} \nu^{\prime}}^{\lambda}$ functions are

$$
\begin{aligned}
& f_{11}^{1}=\left(\frac{4 \xi^{1} \xi^{2}-\xi^{3} d^{7}}{d^{1} d^{2}}\right)^{2}, \\
& f_{11}^{2}=\left(\frac{4 \xi^{1} \xi^{3}+\xi^{2} d^{7}}{d^{1} d^{2}}\right)^{2},
\end{aligned}
$$

$$
f_{12}^{1}=f_{21}^{1}=-f_{12}^{2}=-f_{21}^{2}=\frac{8\left(4 \xi^{1} \xi^{2}-\xi^{3} d^{7}\right)\left(4 \xi^{1} \xi^{3}+\xi^{2} d^{7}\right)}{\left(d^{6}\right)^{2} d^{1} d^{2}}
$$

$$
f_{22}^{1}=\frac{64\left(4 \xi^{1} \xi^{3}+\xi^{2} d^{7}\right)}{\left(d^{6}\right)^{4}}
$$

$$
f_{22}^{2}=\frac{64\left(4 \xi^{1} \xi^{2}-\xi^{3} d^{7}\right)}{\left(d^{6}\right)^{4}}
$$

$f_{22}^{3}=\left[\frac{2 d^{4}}{\left(d^{6}\right)^{2}}-1\right]^{2}$

$f_{23}^{3}=f_{32}^{3}=\frac{2 d^{4}}{\left(d^{6}\right)^{2}}-1$

$$
f_{33}^{3}=1
$$

Clearly, $g_{\mu \nu}$ is analytical, as are its gradient and its Hessian; however, the analytical expressions for these as functions of 


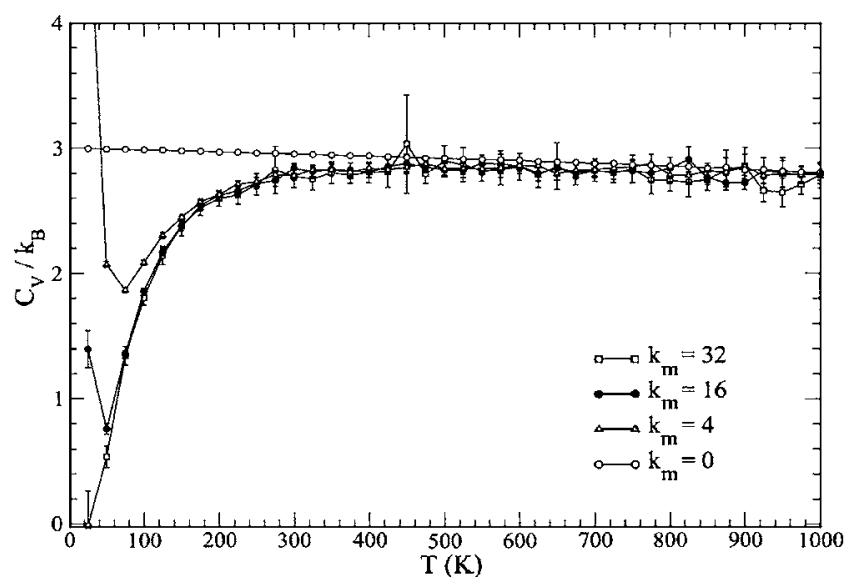

FIG. 6. The finite $k_{m}$ heat capacity of a rigid water molecule in the center of mass frame with hindered rotations.

$\xi^{\nu}$ are formidable. Therefore, it is simpler to compute $f_{\mu^{\prime} \nu^{\prime}}^{\lambda}$ and $J_{\mu}^{\mu^{\prime}}$ and translate the sum in Eq. (41) directly into code.

The eigenvalues of the inertia tensor $I_{\lambda}$ are chosen to be identical to those of rigid water, namely, $I_{x}, I_{y}, I_{z} \approx 12614$, 8588,4026 a.u., respectively. The rotations are hindered using an external scalar field represented by the function

$$
V\left(\xi^{1}, \xi^{2}, \xi^{3}\right)=-V_{0} \frac{\xi^{1}+\xi^{2}+\xi^{3}}{\left(\xi^{1}\right)^{2}+\left(\xi^{2}\right)^{2}+\left(\xi^{3}\right)^{2}+4} .
$$

The value of $V_{0}$ is 0.04 hartree. $V_{0}$ is in the same order of magnitude of the cohesive energy of a water molecule in the bulk. $V_{0}$ is about six times larger than the binding energy of the water dimer and about two times larger than the typical cohesive energy of a water molecule in the octamer. The heat capacity of the system at various temperatures is presented in Fig. 6 for a number of values of core path variables $k_{m}$. The simulations are carried out between 20 and $1000 \mathrm{~K}$, in regular increments of $20 \mathrm{~K}$ using Metropolis sampling. The heat capacity is estimated using the RRS with $k_{m}^{\prime}=4 k_{m}$ and the numerical derivative estimator in Eq. (29). A total of 42 independent samples are collected at every temperature using the Metropolis algorithm. Each sample consists of $1 \times 10^{6}$ moves used to reach the asymptotic distribution, followed by $1 \times 10^{6}$ moves over which the potential energy, the total energy, and the heat capacity are collected. Each move consists of a configuration change $\xi^{1}, \xi^{2}, \xi^{3} \rightarrow\left(\xi^{1}, \xi^{2}, \xi^{3}\right)^{\prime}$ and the translation of a randomly selected path variable for each coordinate $a_{k}^{1}, a_{k}^{2}, a_{k}^{3} \rightarrow\left(a_{k}^{1}, a_{k}^{2}, a_{k}^{3}\right)^{\prime} . k$ is an integer selected using a uniform discrete distribution in $\left(1 \leqslant k \leqslant k_{m}^{\prime}\right)$. The data graphed in Fig. 6 have a number of reassuring features. The $k_{m}=0$ curve is the classical estimate of the heat capacity. The $k_{m}=0$ value of $C_{v} / k_{B}$ clearly approaches the proper equipartition value. The classical value of $C_{v} / k_{B}$ decreases systematically as the temperature increases, indicating that the hindered rotations gradually develop a free character at high temperatures. The heat capacity estimators for $k_{m}>0$ behave very well statistically and show a clear convergence pattern that one anticipates for such a system. All the $k_{m}>0$ data merge into the classical data at high temperatures. The system behaves classically only above $500 \mathrm{~K}$. This temperature is large compared to the freezing and boiling temperature of bulk water. The convergence information gathered with this simpler model is indicative of the convergence pattern that a bulk water simulation may display. The $k_{m}=4$ simulation is converged for $T>260 \mathrm{~K}$. The $k_{m}=16$ simulation converges for $T>80 \mathrm{~K}$. The $k_{m}=32$ simulation is probably converged for $T>60 \mathrm{~K}$; a cluster of rigid water molecules may require relatively larger values of $k_{m}$ to converge at these temperatures.

\section{Simulations of water clusters}

\section{The manifold for $\boldsymbol{n}$ asymmetric rigid tops}

Let $q^{\mu}$ represent the $6 n$-dimensional vector associated with a configuration point in the space $\left(\mathbb{R}^{3} \otimes I^{3}\right) \otimes\left(\mathbb{R}^{3}\right.$ $\left.\otimes \mathbb{I}^{3}\right) \cdots\left(\mathbb{R}^{3} \otimes \mathbb{I}^{3}\right)$, where $\otimes$ is the Cartesian product, $R^{3}$ is the three-dimensional Euclidean space for the center of mass of a top, and $\mathbb{I}^{3}$ symbolizes the inertia ellipsoid, the configuration space for the orientations. In deriving the algorithms and for the implementations of our code, we use the following ordering for the coordinates: $q^{6(i-1)+1}, q^{6(i-1)+2}$, and $q^{6(i-1)+3}$ $(1 \leqslant i \leqslant n)$ represent, respectively, the $x, y$, and $z$ coordinates of the center of mass for the $i$ th top; $q^{6(i-1)+4}, q^{6(i-1)+5}$, and $q^{6 i}(1 \leqslant i \leqslant n)$ represent the three stereographic projection coordinates for the $i$ th top. With these definitions the metric tensor takes the following block-diagonal form:

$$
g_{\mu \nu}=\left(\begin{array}{ccc}
\mathbf{g}^{(1)} & 0 & 0 \\
\cdots & \ldots & \cdots \\
0 & 0 & \mathbf{g}^{(n)}
\end{array}\right) .
$$

The molecular metric tensor $\mathbf{g}^{(i)}$ is represented by a $6 \times 6$ block-diagonal matrix,

$$
\mathbf{g}^{(i)}=\left(\begin{array}{ccc|c}
m & 0 & 0 & \\
0 & m & 0 & \mathbf{0} \\
0 & 0 & m & \\
\hline & \mathbf{0} & & \mathbf{G}^{(i)}
\end{array}\right),
$$

with $m$ representing the mass of the molecular top and $\mathbf{G}^{(i)}$ representing the metric tensor associated with the orientations. The six independent elements of $\mathbf{G}^{(i)}$ are calculated using Eq. (41). Given the sparse nature and block-diagonal structure of $g_{\mu \nu}$, it is not difficult to design algorithms that only require $O(n)$ operations for the computation of $g_{\mu \nu} \dot{q}^{\mu} \dot{q}^{\nu}$,

$$
g_{\mu \nu} \dot{q}^{\mu} \dot{q}^{\nu}=\sum_{i=1}^{n} g_{\rho \lambda}^{(i)} \dot{q}^{6(i-1)+\rho} \dot{q}^{6(i-1)+\lambda} .
$$

The evaluation of $g_{\mu \nu} \dot{q}^{\mu} \dot{q}^{\nu}$ requires CPU times comparable to those needed for the computation of an empirical potential surface in the equidimensional space. Analytical expressions for the Hessian elements of $g_{\mu \nu}$ can be obtained. However, the computation of the Hessian of $g_{\mu \nu}$ is just as expensive as the Hessian of a typical empirical potential surface of equal dimension. Therefore, the present formulation of the RRS method in curved spaces is considerably cheaper than the previous one.

It is important to clarify that our use of the space $\left(\mathbb{R}^{3} \otimes \mathbb{I}^{3}\right) \otimes\left(\mathbb{R}^{3} \otimes \mathbb{I}^{3}\right) \cdots\left(\mathbb{R}^{3} \otimes \mathbb{I}^{3}\right)$ does not introduce bias in the distribution function of the constrained system. In 


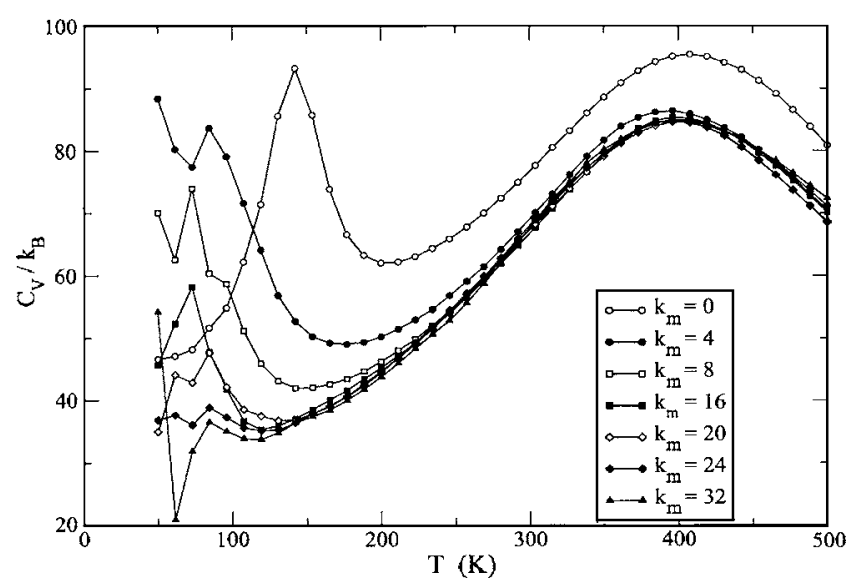

FIG. 7. The heat capacity of the water octamer for various values of $k_{m}$ [Eq. (29)] showing the convergence profile of the estimator.

Ref. 49, the authors carefully analyze the difference between the use of simulations in constrained spaces compared to those carried out in the Euclidean space mapped with internal coordinates and with infinitely stiff springs for the high frequency modes. The example of the bending angle distribution on a trimer is detailed in Ref. 49. The authors suggest including a $\left(g_{E} / g\right)^{1 / 2}$ term in classical simulations, where $g_{E}$ is the determinant of the metric tensor of the Euclidean space mapped with internal coordinates and $g$ is the same for the curved space. However, it can be shown in general, for both linear and nonlinear tops, that $\left(g_{E} / g\right)^{1 / 2}$ is both translationally and rotationally invariant even when the orientations are mapped projectively as we do presently. ${ }^{51}$ Therefore, while it is possible to include such correction in PIMC simulations in curved spaces, we ignore this additional complication in the present work since a constant term cannot affect the dynamics.

\section{Heat capacities for the trimer through the octamer}

In Figs. 7 and 8 we present the results of several simulations of water clusters, with each molecule modeled as a rigid top. The data sets are computed using 20 independent move blocks in the non-Euclidean manifold $\left(\mathbb{R}^{3} \otimes \mathbb{I}^{3}\right) \otimes\left(\mathbb{R}^{3}\right.$ $\left.\otimes \mathbb{I}^{3}\right) \cdots\left(\mathbb{R}^{3} \otimes \mathbb{I}^{3}\right)$. Each block consists of $1 \times 10^{6}$ warmup moves followed by $1 \times 10^{6}$ simulation moves. The standard error in the mean is used to estimate the $95 \%$ confidence interval. The confidence interval is represented graphically by error bars in Fig. 8. Each move consists of a translation or a rotation of a randomly selected molecule, and a translation of one path variable for each coordinate, e.g., $a_{k}^{6(i-1)+1}$, $a_{k}^{6(i-1)+2}, a_{k}^{6(i-1)+3} \rightarrow\left(a_{k}^{6(i-1)+1}, a_{k}^{6(i-1)+2}, a_{k}^{6(i-1)+3}\right)$. The integer $k$ is in $\left(1 \leqslant k \leqslant k_{m}^{\prime}\right)$ and is selected randomly from a uniform discrete distribution in the set. The simulations employ parallel tempering with 40 walkers at evenly spaced temperatures between 50 and $500 \mathrm{~K}$. To define the cluster, a constant volume continuous Lee-Barker-Abraham spherical cavity is employed,

$$
V_{\mathrm{LBA}}=\sum_{i=1}^{8}\left(\frac{\mathbf{r}_{\mathrm{O}_{i}}-\mathbf{R}_{\mathrm{CM}}}{2 R_{C}}\right)^{20},
$$

where $\mathbf{r}_{\mathrm{O}_{i}}$ is the location of the oxygen atom for the $i$ th water molecule, $\mathbf{R}_{\mathrm{CM}}$ is the center of mass of the cluster, and $R_{C}$ is 9.0 bohr.

The $k_{m}=24$ graph is in the uniform convergence asymptote above $100 \mathrm{~K}$, while the heat capacity is still converging nonuniformly below $100 \mathrm{~K}$ with $k_{m}=24$ core coefficients. The data sets in Fig. 7 are graphed without error bars to make the graphs less cluttered and to make the convergence pattern stand out. The quantum heat capacities in Fig. 8 are computed with $k_{m}=24$, which are converged within the statistical fluctuations for $T \geqslant 100 \mathrm{~K}$ for the octamer. The difference between the classical and the quantum simulations remains substantial at $500 \mathrm{~K}$ for all sizes; nevertheless, it is clear in both Figs. 7 and 8 that the two estimates converge at some higher $T$.

\section{Characterization of the random walks for $\left(\mathrm{H}_{2} \mathrm{O}\right)_{8}$}

Given the large discrepancies between the classical and the quantum simulations, we explore the simple point charge (SPC) potential energy surface for the dimer through the octamer to find all the important minima; in turn these are used
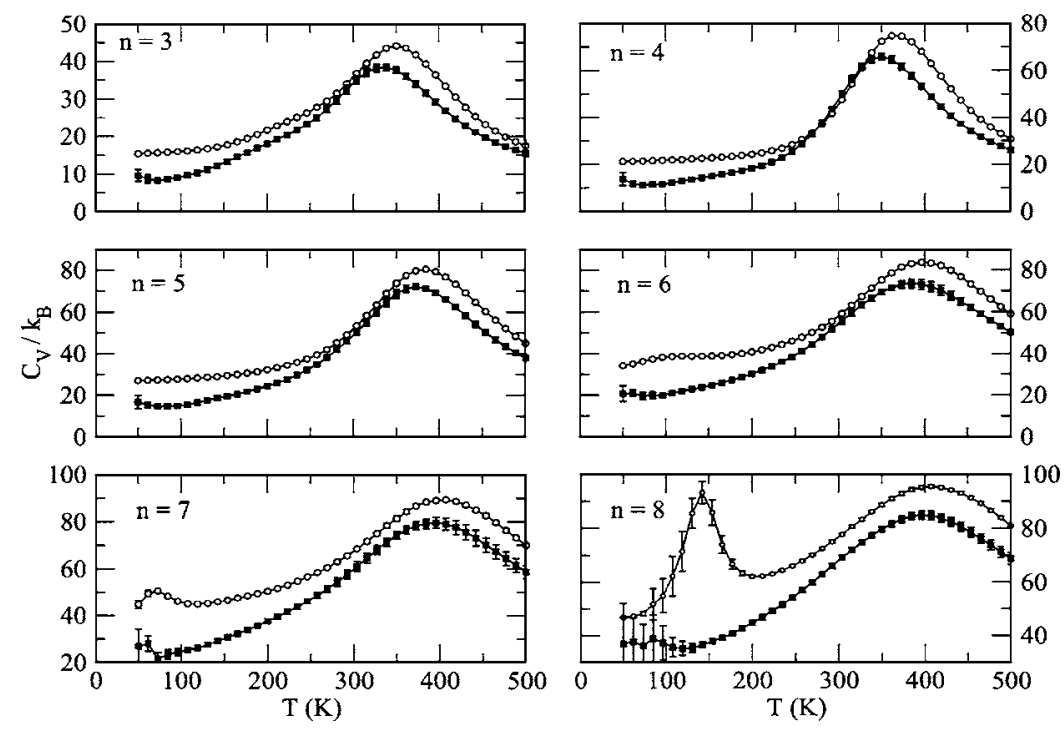

FIG. 8. The classical (white circles) and quantum $\left(k_{m}\right.$ $=24$, black squares) heat capacity of water clusters obtained with the RRS method (Sec. II) and the finite difference estimator in Eq. (29). 
to characterize the random walks using a Structural Comparison Algorithm (SCA). ${ }^{52}$ The quantum effects are most conspicuous for the octamer, for which the pronounced melting feature in the classical simulation is "washed away" by quantum fluctuations. Therefore, as a check, we minimize all the sizes from the dimer through the octamer and compare our minima with those found by other groups with similar potentials. ${ }^{50,53-58}$ We use the minima of the octamer to characterize the configurations in several random walks. To minimize the structures, we use the full $R^{9 n}$ space mapped by Cartesian coordinates, with the potential along the covalent stretching and bending degrees of freedom obtained with the F2 extension. ${ }^{59}$ The candidate structures are generated with the genetic algorithm, and these are quenched with a $T=0$ Brownian dynamics algorithm, which in Euclidean spaces with Cartesian coordinates takes a simple form,

$$
\ddot{x}^{\mu}+\delta^{\mu \nu} \partial_{\nu} V+\gamma x^{\mu}=0 .
$$

Here, $\gamma$ is the coefficient of friction. The genetic algorithm is implemented to create 100 candidate structures using simple slicing-rejoining operators. The candidates are quenched, sorted by energy in ascending order, and compared with SCA if the energy difference between two minima is less than $1 \times 10^{-4}$ hartree.

SCA provides us with a measure of distance between two configurations that are being compared. This comparison is obtained by attempting to find the best superposition of two structures through a set of translations, rotations, and a sorting process. Typically a running configuration is compared with a minimum. In the case of the genetic algorithm, we compare the newly quenched candidates with all the minima already known. This eliminates multiple copies of a minimum included in the genetic pool that one uses for the next generation of "children structures." The key difference with out implementation is that the comparison process allows both enantiomers of optically active minima to contribute to the "gene pool." Many minima lack an improper rotation axis and consequently display optical isomerism. If all the degenerate replicas of an optically active minimum are eliminated from the pool, only one of the two enantiomers is used in the generation of new candidates. The impact of eliminating enantiomers from the gene pool could have on the efficiency or even the success of a global optimization is not known, but one can anticipate that when a large number of atoms are involved this detail may become important. We run 40 generations for the hexamer, heptamer, and octamer. However, the global minimum for all the sizes from the dimer through the octamer is found very quickly by the genetic algorithm.

The structures we find for the global minima agree qualitatively with those reported in a number of global minimization studies carried out with related potential functions. ${ }^{50,53-58}$ In particular, we find all the cubic isomers reported recently by Nigra et al. ${ }^{50}$ In agreement with Nigra et al. ${ }^{50}$ we find that the $D_{2 d}$ cube is lower in energy than the $S_{4}$ cube; we find the $C_{2}, C_{i}$, and five $C_{1}$ cubes with slightly different energetic orders. We focus our comparison on Nigra et $a .^{50}$ since the authors report a characterization of the classical parallel tempering walk similar to the one we carry out.
The $C_{2}, C_{i}$, and $C_{1}$ cubes have energies comparable to other open minima of the octamer potential energy surface and contribute only slightly in the melting range. Our melting peak in the classical calculation is at $T_{\max }=142.3 \mathrm{~K}$ instead of the $T_{\max }=178.5 \mathrm{~K}$ reported by Nigra et al. The potential used by the authors of Ref. 50 is a sophisticated model, ${ }^{53}$ known as the Matsuoka-Clementi-Yoshimine (MCY) potential, constructed by fitting CI computations on the water dimer, and is quite different from the SPC model we use here; discrepancies are to be expected. Therefore, it is rewarding to find that the characterization of the walks generated on the SPC surface that we report in the following is in excellent qualitative agreement with the results obtained by the MCY surface.

We use SCA to characterize the walk in the following manner. Let $\mathbf{x}_{A}^{i}$ represent the Cartesian coordinates of atom $i$ in a configuration under analysis after a systematic set of translation, rotations, and permutations is carried out to find the best possible overlap with the global minimum configuration. $\left\{\mathbf{x}_{0}^{i}\right\}_{i=1}^{3 n}$ represents the Cartesian coordinates of the atoms in the global minimum. Then,

$$
\Delta_{0}=\sum_{i=1}^{3 n}\left|\mathbf{x}_{A}^{i}-\mathbf{x}_{0}^{i}\right|
$$

represents the "structural distance" from the global minimum for $\left\{\mathbf{x}_{A}^{i}\right\}_{i=1}^{3 n}$. Histograms of $\Delta_{0}$ can be used to detect features in the density matrix arising from as solid-solid transformations, melting, and similar thermally induced phenomena. Figure 9 contains two sets of such histograms for $\Delta_{0}$ sampled in the $T$ interval below the melting peak of the classical heat capacity. The graphs in Fig. 9(a) are obtained by measuring $10^{5}$ configurations during the classical walk after $1 \times 10^{6}$ warmup moves. The graphs in Fig. 9(b) are obtained with $k_{m}=24$. The broad feature between 10 and 40 bohrs in both figures is comprised of cubic structures; these are either the $D_{2 d}$ or $S_{4}$ isomer. The $S_{4}$ isomer produces a shoulder of this feature above 30 bohrs. In the classical simulation [Fig. 9(a)] this shoulder is detectable at $50 \mathrm{~K}$ and gradually gains intensity. The first detected configurational jump for a cube to one of the open structures (typically intersecting rings) in the classical simulation is at $96.2 \mathrm{~K}$. This peak in the histograms of $\Delta_{0}$ is well differentiated from the broad features associated with cubic structures.

The isomeric distributions we measure in the classical simulation agree qualitatively with those reported by Nigra et al. $^{50}$ in a number of key features. We see a significant $D_{2 d} \rightarrow S_{4}$ isomerization present at $50 \mathrm{~K}$. Nigra et al. reported the onset of $D_{2 d} \rightarrow S_{4}$ isomerizations at $40 \mathrm{~K}$. Similarly, we find that the other cubic structures (the $C_{2}, C_{i}$, and $C_{1}$ cubes) play a small role in the liquid region. This finding agrees with the reported probabilities for this group of structures. In Fig. 8 of Ref. 50, Nigra et al. reported the onset of liquid structures above $120 \mathrm{~K}$. We find the onset of liquid structures around $96 \mathrm{~K}$, explaining the difference in the peak temperature for the melting.

Probably, the quantum distributions of $\Delta_{0}$ [Fig. 9(b)] are not converged at $73.1 \mathrm{~K}$ and below. The abrupt appearance of the feature at 75 bohrs at $73.1 \mathrm{~K}$ is suggestive of the lack 


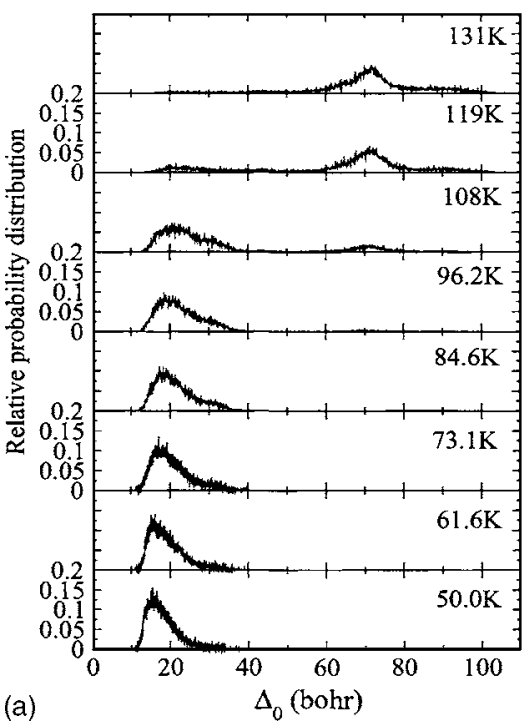

(a)

of convergence for the two distributions of $\Delta_{0}$ at 61.6 and 50.0 K. Nevertheless, we can see a pattern developing, even at the colder temperatures. The $D_{2 d} \rightarrow S_{4}$ equilibrium is shifted to the right considerably compared to the classical simulation. Similarly, the cube $\rightarrow$ liquid phase change is shifted. At $84.6 \mathrm{~K}$ we can say with confidence that the octamer is melting. However, the quantum distributions of $\Delta_{0}$ continue to display distinguishable albeit broad features at higher temperatures. The feature centered at 40 bohrs that grows systematically at 40 bohrs between 96.2 and $119 \mathrm{~K}$ contains a mixture of $S_{4}$ and $C_{2}, C_{i}$, and $C_{1}$ cubes, whereas the feature that grows around 60 bohrs from $84.6 \mathrm{~K}$ contains cubic structures with a significant amount of obliqueness and cubic structures with one missing vertex. The remarkable differences in the distributions of $\Delta_{0}$ between classical and quantum simulations explain the large differences visible in the thermodynamic properties. One would anticipate that quantum fluctuations would simply broaden the classical distributions of $\Delta_{0}$ and shift isomerization features to lower temperatures. That shifting and broadening can be gleaned in Fig. 9. However, one would not anticipate the appearance of new features that remain well resolved at elevated temperatures inside the liquid coexistance region. In fact, this has been observed recently in other hydrogen bonded systems. ${ }^{42}$

\section{CONCLUSIONS}

The two main objectives for the work reported in this article are to illustrate the efficiency gained by using holonomic constraints in PIMC simulations of molecular condensed matter and the exploration of the quantum thermodynamic properties of small water clusters.

It is well known that rigid body simulations improve the efficiency of thermodynamic estimators in Metropolis simulations; however, little is known about how holonomic constraints affect the convergence of PIMC simulations. We have investigated a simple one-dimensional harmonic chain using analytical solutions of the thermodynamic path integral for finite Trotter number. For this system the analytical solutions of the path integral expression for the heat capacity as a function of $T$ and the Trotter number (or a number of path variables) clearly support our hypothesis: The choice to use holonomic constraints in PIMC simulations is dictated by the efficiency of a simulation as it relates to convergence properties rather than statistical fluctuations. The present investigation shows that if the smallest frequency in the intramolecular set is at least a factor of 10 greater than the largest frequency in the intermolecular set, then the adiabatic approximation is not only convenient; it is necessary to produce numerical convergence with reasonable means at low temperatures. The numerical tests with the harmonic chain reveal that the primitive algorithm simulation in the adiabatic limit converges better than the cubically convergent estimator for the flexible case. The fully flexible Cartesian simulation becomes unfeasible at low temperatures, where the thermodynamics are dominated by intermolecular rearrangements and the "molecules" are predominantly in the ground vibrational state. Since the results obtained from the harmonic chain and the conclusions that can be drawn are for 1000 point masses, there is evidence that these results may apply to bulk molecular condensed matter as well as clusters.

The graphs in Fig. 4(a), however, point to the next challenge for the present developments in the statistical thermodynamic theory of condensed molecular matter. Clearly, between $1<T^{*}<2$ the system does not behave classically, and the adiabatic approximation is not satisfactory. Figure 4 suggests that perhaps one only needs to perform a flexible PIMC simulation for $T^{*}>1$ and a rigid simulation below this value. However, in more complex models of condensed matter there may be more than one high frequency mode. Furthermore, even the rigid simulation may require parallel tempering runners at temperatures above $T^{*}=1$ to overcome potential energy barriers. These complications arise in the quantum simulations of water clusters. With the parameters of the SPC/F2 in Lobaugh and Voth, ${ }^{59}$ one obtains the following frequencies for water. The fundamental for the $\mathrm{H}-\mathrm{O}-\mathrm{H}$ bend is at $1504 \mathrm{~cm}^{-1}$, the $\mathrm{O}-\mathrm{H}$ symmetric stretch is at $3322 \mathrm{~cm}^{-1}$, and the asymmetric $\mathrm{O}-\mathrm{H}$ stretch is at $3601 \mathrm{~cm}^{-1}$. These frequencies are substantially redshifted from the gas phase data since the SPC/F2 has been designed for molecular dynamics simulations of bulk water. These fre- 
quencies are likely to be only rough approximations for a small water cluster such as the octamer. Nevertheless, with these data, and with $T^{*}=(\beta \hbar \omega)^{-1}$ and $T \approx(1.520 \mathrm{~K} \mathrm{~cm}) \widetilde{\nu} T^{*}$, we can examine some characteristic temperatures associated with the intramolecular vibrations in the water clusters. Assuming that for $T^{*}<0.1$ the harmonic oscillator is predominantly in the ground state, and that for $T^{*}>2$ it behaves classically, one concludes that the $\mathrm{H}-\mathrm{O}-\mathrm{H}$ bending modes are essentially in the ground state for $T<230 \mathrm{~K}$, the $\mathrm{H}-\mathrm{O}$ symmetric stretches are essentially in the ground state for $T<505 \mathrm{~K}$, and the $\mathrm{H}-\mathrm{O}$ asymmetric stretches are predominantly in the ground state for $T<550 \mathrm{~K}$. Below $550 \mathrm{~K}$, a flexible calculation has to converge the density matrix along the stretching modes to a distribution dominated by the ground state wave functions, and the convergence of estimators is highly nonuniform. Therefore, in the $100-230 \mathrm{~K}$, the stereographic projection path integral employed here is much more efficient than its flexible equivalent with Cartesian coordinates. In the $230-500 \mathrm{~K}$ range, the best model should allow bending, but the stretches should be constrained. At present, the space metric for the seven-dimensional space which includes the translation of the center of mass, the three rotations, and the bending mapped by stereographic projections awaits development; this is the future direction of our research. The resulting algorithm should be suitable for simulations in the $230-500 \mathrm{~K}$ range, not only in clusters but in bulk water, with much greater efficiency than was previously possible.

\section{ACKNOWLEDGMENTS}

One of the authors (E.C.) acknowledges Professor P. Campbell for many long constructive conversations surrounding water clusters. This work has been supported by the National Science Foundation (Grant No. CHE0554922). Additionally, E.C. acknowledges the donors of the Petroleum Research Fund, administered by the ACS (Grant No. 40946B6), The Stacy Ann Vitetta '82 Professorship Fund and The Ellington Beavers Fund for Intellectual Inquiry from Arcadia University for partial support of this research.

\section{APPENDIX: TRANSFORMING FROM EULER ANGLES TO CARTESIAN COORDINATES AND BACK}

Most empirical intermolecular potential energy surface models are relatively simple expressions of the Cartesian coordinates for each atom. Consequently, it is important to find the Cartesian coordinates of all the atoms in the molecular top $i$ when the center of mass and the three stereographic projections are known. There are two ways to convert the Cartesian coordinates of all atoms to Euler angles, and then to stereographic projections, each yielding equivalent metrics in the inertia ellipsoid. The active map of the body fixed frame is considered in this Appendix. The transformation of the passive map can be handled in a similar way. The Euler angles can be obtained from the stereographic projections by using Eqs. (42)-(44). The Cartesian coordinates of three points in a rigid body in the center of mass coordinate system can be obtained from the three Euler angles once a reference configuration in the body frame common to all tops is specified. For water clusters we take the following reference configuration:

$$
\begin{array}{cccc} 
& x & y & z \\
\mathrm{H}_{1} & 0 & \alpha_{y} & \gamma_{z} \\
\mathrm{O} & 0 & \beta_{y} & 0 \\
\mathrm{H}_{2} & 0 & \alpha_{y} & -\gamma_{z}
\end{array},
$$

where for the rigid model of water $\alpha_{y}=0.9865, \beta_{y}$ $=-0.1243$, and $\gamma_{z}=1.529$ bohrs. The reference configuration defines the space axes; this is a configuration point in which the inertia tensor is diagonal. These space axes can be obtained by simply transforming back from the reference body frame to the running configuration, and the inverse mapping can be obtained as well. The problem can be stated as follows. Let the element $\mathbf{R} \in \mathrm{O}(3)$ be the rotation matrix that takes the reference configuration to an arbitrary running configuration (namely, the body coordinates in the center of mass Cartesian space). When expressed in terms of Euler angles the representation of the element $\mathbf{R} \in \mathrm{O}(3)$ is as follows: ${ }^{60}$

$$
\mathbf{R}=\left(\begin{array}{ccc}
\cos \phi \cos \psi-\cos \theta \sin \phi \sin \psi & \sin \phi \cos \psi+\cos \theta \cos \phi \sin \psi & \sin \psi \sin \theta \\
-\cos \phi \sin \psi-\cos \theta \sin \phi \cos \psi & -\sin \phi \sin \psi+\cos \theta \cos \phi \cos \psi & \cos \psi \sin \theta \\
\sin \phi \sin \theta & -\cos \phi \sin \theta & \cos \theta
\end{array}\right)
$$

This definition yields nine equivalences between the Eulerian angles and the Cartesian coordinates of each atom; from these it is straightforward to derive the following relationships between the trigonometric functions and the center of mass coordinates:

$$
\cos \theta=\frac{z_{\mathrm{H}_{1}}-z_{\mathrm{H}_{2}}}{2 \gamma_{z}},
$$

$$
\begin{aligned}
& \sin \theta=\frac{1}{2 \gamma_{z}} \sqrt{\left(x_{\mathrm{H}_{1}}-x_{\mathrm{H}_{2}}\right)^{2}+\left(y_{\mathrm{H}_{1}}-y_{\mathrm{H}_{2}}\right)^{2}}, \\
& \sin \psi=\frac{x_{\mathrm{H}_{1}}-x_{\mathrm{H}_{2}}}{2 \gamma_{z} \sin \theta}, \\
& \cos \psi=\frac{y_{\mathrm{H}_{1}}-y_{\mathrm{H}_{2}}}{2 \gamma_{z} \sin \theta},
\end{aligned}
$$




$$
\begin{aligned}
& \cos \phi=-\frac{z_{\mathrm{O}}}{\beta_{y} \sin \theta}, \\
& \sin \phi=\frac{1}{\cos \psi}\left(\frac{x_{\mathrm{H}_{1}}+x_{\mathrm{H}_{2}}}{2 \alpha}-\cos \theta \cos \phi \sin \psi\right) .
\end{aligned}
$$

Some of these equations become infinite for those special cases (rare events in random walks), where $\sin \theta=0$. Two possibilities must be considered:

Case 1: $\theta=0$, The rotation matrix becomes

$$
\mathbf{R}=\left(\begin{array}{ccc}
\cos (\phi+\psi) & \sin (\phi+\psi) & 0 \\
-\sin (\phi+\psi) & \cos (\phi+\psi) & 0 \\
0 & 0 & 1
\end{array}\right) .
$$

The body coordinates now are

$$
\begin{aligned}
& x_{\mathrm{H}_{1}}=x_{\mathrm{H}_{2}}=\alpha_{y} \sin (\phi+\psi), \\
& x_{\mathrm{O}}=\beta_{y} \sin (\phi+\psi), \\
& y_{\mathrm{H}_{1}}=y_{\mathrm{H}_{2}}=\alpha_{y} \cos (\phi+\psi), \\
& y_{\mathrm{O}}=\beta_{y} \cos (\phi+\psi), \\
& z_{\mathrm{H}_{1}}=-z_{\mathrm{H}_{2}}=\gamma_{z} ; \quad z_{\mathrm{O}}=0 .
\end{aligned}
$$

Case 2: $\theta=\pi$. Then, the element of $\mathrm{O}(3)$ is

$$
\mathbf{R}=\left(\begin{array}{ccc}
\cos (\phi-\psi) & \sin (\phi-\psi) & 0 \\
\sin (\phi-\psi) & -\cos (\phi-\psi) & 0 \\
0 & 0 & -1
\end{array}\right)
$$

The center of mass space fixed frame now gives the following coordinates for the atoms:

$$
\begin{aligned}
& x_{\mathrm{H}_{1}}=x_{\mathrm{H}_{2}}=\alpha_{y} \sin (\phi-\psi), \\
& x_{\mathrm{O}}=\beta_{y} \sin (\phi-\psi), \\
& y_{\mathrm{H}_{1}}=y_{\mathrm{H}_{2}}=-\alpha_{y} \cos (\phi-\psi), \\
& y_{\mathrm{O}}=-\beta_{y} \cos (\phi-\psi), \\
& z_{\mathrm{H}_{1}}=-z_{\mathrm{H}_{2}}=-\gamma_{z}, \quad z_{\mathrm{O}}=0 .
\end{aligned}
$$

Both special cases can be considered without loss of generality as a single rotation about the space $z$ axis by $\phi$ radians. Therefore, one arrives at

$$
\begin{aligned}
& \sin \phi=x_{\mathrm{H}_{1}} / \alpha_{y}, \\
& \cos \phi= \pm y_{\mathrm{H}_{1}} / \alpha_{y}
\end{aligned}
$$

for the $\theta=0$ and $\pi$, respectively. A translation of the center of mass for each top completes the transformation from the coordinates of the center of mass-projection space to the full Euclidean space $\left(\mathbb{R}^{3} \otimes \mathbb{I}^{3}\right) \otimes\left(\mathbb{R}^{3} \otimes I^{3}\right) \cdots\left(\mathbb{R}^{3} \otimes I^{3}\right) \rightarrow \mathbb{R}^{9 n}$ which contains the Cartesian coordinates of each atom.
${ }^{1}$ R. P. Feynman, Rev. Mod. Phys. 20, 367 (1948).

${ }^{2}$ B. S. DeWitt, Rev. Mod. Phys. 29, 377 (1957).

${ }^{3}$ L. S. Shulman, Techniques and Applications of Path Integration (Wiley, New York, 1981).

${ }^{4}$ J. D. Doll, D. L. Freeman, and T. L. Beck, Adv. Chem. Phys. 78, 61 (1990).

${ }^{5}$ D. L. Freeman and J. D. Doll, Adv. Chem. Phys. 70B, 139 (1998).

${ }^{6}$ R. D. Coalson, J. Chem. Phys. 85, 926 (1986).

${ }^{7}$ A. E. Cho, J. D. Doll, and D. L. Freeman, J. Chem. Phys. 117, 5971 (2002).

${ }^{8}$ D. M. Ceperley, Rev. Mod. Phys. 67, 279 (1995).

${ }^{9}$ B. J. Berne and D. Thirumalai, Annu. Rev. Phys. Chem. 37, 401 (1986).

${ }^{10}$ D. Kim, J. D. Doll, and D. L. Freeman, J. Chem. Phys. 108, 3871 (1998).

${ }^{11}$ B. Chen, M. A. Gomez, J. D. Doll, and D. L. Freeman, J. Chem. Phys. 108, 4031 (1998).

${ }^{12}$ J. P. Neirotti, D. L. Freeman, and J. D. Doll, J. Chem. Phys. 112, 3990 (2000).

${ }^{13}$ K. R. Glaesemann and L. E. Fried, J. Chem. Phys. 117, 3020 (2002).

${ }^{14}$ R. D. Coalson, D. L. Freeman, and J. D. Doll, J. Chem. Phys. 85, 4567 (1986).

${ }^{15}$ M. Eleftheriou, J. D. Doll, E. Curotto, and D. L. Freeman, J. Chem. Phys. 110, 6657 (1999).

${ }^{16}$ C. Predescu, J. D. Doll, and D. L. Freeman, e-print cond-mat/0301525.

${ }^{17}$ S. L. Mielke, J. Srinivasan, and D. G. Truhlar, J. Chem. Phys. 112, 8758 (2000).

${ }^{18}$ S. L. Mielke and D. G. Truhlar, J. Chem. Phys. 114, 621 (2001).

${ }^{19}$ C. Predescu, e-print cond-mat/0302171.

${ }^{20}$ C. Predescu, D. Sabo, and J. D. Doll, J. Chem. Phys. 119, 4641 (2003).

${ }^{21}$ C. Predescu and J. D. Doll, J. Chem. Phys. 117, 7448 (2002).

${ }^{22}$ C. Predescu, D. Sabo, J. D. Doll, and D. L. Freeman, J. Chem. Phys. 119, 10475 (2003)

${ }^{23}$ C. Predescu, D. Sabo, J. D. Doll, and D. L. Freeman, J. Chem. Phys. 119, 12119 (2003)

${ }^{24}$ D. Sabo, C. Predescu, and J. D. Doll, J. Chem. Phys. 121, 856 (2004).

${ }^{25}$ C. Predescu, P. A. Frantsuzov, and V. A. Mandelshtam, J. Chem. Phys. 122, 154305 (2005).

${ }^{26}$ B. Schutz, A First Course in General Relativity (Cambridge, New York, 1985).

${ }^{27}$ W. D. Curtis and F. R. Miller, Differential Manifolds and Theoretical Physics (Academic, New York, 1985).

${ }^{28}$ H. Kleinert, Path Integrals in Quantum Mechanics, Statistics and Polymer Physics (World Scientific, Singapore, 1990).

${ }^{29}$ R. A. Kuharski and P. J. Rossky, J. Chem. Phys. 82, 5164 (1985).

${ }^{30}$ D. Marx and M. H. Müser, J. Phys.: Condens. Matter 11, R117 (1999).

${ }^{31}$ D. Marx and P. Nielaba, Phys. Rev. A 45, 8968 (1992).

${ }^{32}$ N. Blinov and P.-N. Roy, J. Chem. Phys. 120, 3759 (2004).

${ }^{33}$ N. Blinov, X. Song, and P.-N. Roy, J. Chem. Phys. 120, 5916 (2004).

${ }^{34}$ R. E. Zillich, F. Paesani, Y. Kwon, and K. B. Whaley, J. Chem. Phys. 123, 114301 (2005).

${ }^{35}$ V. Buch, J. Chem. Phys. 100, 7610 (1994).

${ }^{36}$ T. F. Miller III and D. C. Clary, J. Chem. Phys. 119, 68 (2003).

${ }^{37}$ D. C. Clary, J. Chem. Phys. 116, 8262 (2002).

${ }^{38}$ M. F. Russo, Jr. and E. Curotto, J. Chem. Phys. 118, 6806 (2003).

${ }^{39}$ M. F. Russo, Jr. and E. Curotto, J. Chem. Phys. 120, 2110 (2004).

${ }^{40}$ M. W. Avilés and E. Curotto, J. Chem. Phys. 122, 164109 (2005).

${ }^{41}$ E. Curotto, J. Chem. Phys. 123, 134102 (2005).

${ }^{42}$ M. W. Avilés and E. Curotto, J. Chem. Phys. 124, 174305 (2006).

${ }^{43}$ M. Falcioni and M. W. Deem, J. Chem. Phys. 110, 1754 (1999).

${ }^{44}$ J. P. Neirotti, F. Calvo, D. L. Freeman, and J. D. Doll, J. Chem. Phys. 112, 10340 (2000)

${ }^{45}$ F. Calvo, J. P. Neirotti, D. L. Freeman, and J. D. Doll, J. Chem. Phys. 112, 10350 (2000).

${ }^{46}$ J. P. Neirotti, D. L. Freeman, and J. D. Doll, Phys. Rev. E 62, 7445 (2000).

${ }^{47}$ N. Metropolis, A. W. Rosenbluth, M. N. Rosenbluth, A. M. Teller, and E. Teller, J. Chem. Phys. 21, 1087 (1953).

${ }^{48}$ M. P. Allen and D. J. Tildesley, Computer Simulations of Liquids (Claredon, Oxford, 1987).

${ }^{49}$ D. Frenkel and B. Smit, Understanding Molecular Simulations, 2nd ed. (Academic, New York, 1996).

${ }^{50}$ P. A. Nigra, M. C. Cargnano, and S. Kais, J. Chem. Phys. 115, 2621 (2001).

${ }^{51}$ E. Curotto (unpublished). 
${ }^{52}$ M. R. Ghayal and E. Curotto, J. Chem. Phys. 111, 5522 (1999).

${ }^{53}$ H. Matsuoka, E. Clementi, and M. Yoshimine, J. Chem. Phys. 64, 1351 (1976).

${ }^{54}$ H. Kabrede and R. Hentschke, J. Phys. Chem. B 107, 3914 (2003).

${ }^{55}$ R. J. Wawak, M. M. Wimmer, and H. A. Scheraga, J. Phys. Chem. 96, 5138 (1992).
${ }^{56}$ D. J. Wales and I. Ohmine, J. Chem. Phys. 98, 7245 (1993).

${ }^{57}$ B. Hartke, Z. Phys. Chem. 214, 1251 (2000).

${ }^{58}$ I. M. B. Nielsen, E. T. Seidl, and C. L. Janssen, J. Chem. Phys. 110, 9435 (1999).

${ }^{59}$ J. Lobaugh and G. A. Voth, J. Chem. Phys. 106, 2400 (1997).

${ }^{60}$ H. Goldstein, Classical Mechanics (Addison-Wesley, New York, 1965). 
The Journal of Chemical Physics is copyrighted by the American Institute of Physics (AIP). Redistribution of journal material is subject to the AIP online journal license and/or AIP copyright. For more information, see http://ojps.aip.org/jcpo/jcper/jsp 